\title{
Classification of Non-pharmacological Interventions for Managing Pandemics
}

\author{
Peter G. Goldschmidt \\ President, World Development Group, Inc, Bethesda, MD, USA.
}

PGG@WORLDDG.COM

Corresponding Author: Peter G. Goldschmidt.

Copyright (C 2021 Peter G. Goldschmidt This is an open access article distributed under the Creative Commons Attribution License, which permits unrestricted use, distribution, and reproduction in any medium, provided the original work is properly cited.

\begin{abstract}
Pandemics have altered the course of human history. The overarching goal of pandemic response management is to contain pandemogen spread as quickly and as completely as possible; it is not only the first line of defense, it is the only defense. At the start, only non-pharmaceutical interventions (NPI) may be available. There is no classification scheme for NPI. This article 1) proposes both a classification scheme for NPI and a functional way of coding them for descriptive and analytic purposes and 2) by describing the classification scheme, builds an initial inventory of NPI. For classification purposes, NPI can be organized according to the following broad categories: 1) community control, 2) moving and mixing, 3 ) testing and tracing, 4) personal performance, 5) environmental engineering, 6) bodies and burials, and 7) infection interdiction. Classification facilitates describing and analyzing NPI (eg, comparing how countries used different NPI to respond to Covid-19 and to evaluate their effectiveness). Next steps may include 1) elaborating the classification scheme and coding structure in operation detail as an international standard and 2) maintaining a corresponding set of standard definitions. In the interim, any entity could apply the scheme to suit its purposes.
\end{abstract}

Keywords: Classification, Covid-19, Health-informatics, Nomenclature, Non-pharmaceuticalintervention, Ontology, Pandemic, Standard.

\section{INTRODUCTION}

Pandemics have altered the course of human history. Well-known examples (with the greatest impact on human society) include 1) Black Death (1346-1352, which may have killed more than half of the population of Europe), 2) influenza pandemic (1918-1920, which may have killed as many as 100 million people), and 3) Covid-19 (2019-ongoing, which may have already killed more than 18 million people, and still counting) [1,2]. The overarching goal of pandemic response management (PRM) is to contain pandemogen spread as quickly and as completely as possible: preventing spread is not only the first line of defense, it is the only defense [3]. Traditionally, PRM policies and strategies are divided into: 1) non-pharmaceutical interventions (NPI) and 2) pharmaceuticals (vaccines, prophylactics, and treatments). There is no classification scheme for NPI. This article

Citation: Peter G. Goldschmidt. Classification of Non-pharmacological Interventions for Managing Pandemics. Advances in Artificial Intelligence and Machine Learning. 2021;1(3):16. 
1) proposes both a classification scheme and a functional way of coding NPI for descriptive and analytic purposes and 2) by describing the classification scheme, builds an initial inventory of NPI. The scheme classifies NPI used in ancient times, added in modern times, augmented after the 1918 pandemic, and expanded with the Covid-19 pandemic (covid), including digital-age technologies (which may themselves be NPI or may support their implementation). This article does not 1) trace the history of different NPI, 2) assess their practicality or effectiveness, 3) document consequences of NPI use or associated issues (such as fake test certificates), 4) suggest what combination of NPI to employ to control a pandemic based on phase, situation on the ground, or other factor (including nature of pandemogen and chain of transmission), 5) classify techniques for implementing and enforcing NPI nor collateral policies that may be needed to make NPI work (such as income maintenance in relation to stay-at-home orders), nor 6) describe how digital-age technologies have been used to respond to covid or may be used to manage future pandemics.

\section{METHODS}

Developing the NPI classification scheme involved 1) creating a suitable conceptual framework and 2) illustrating it with examples. The framework is based on 1) epidemiological triad and 2) strategies to prevent pandemogen spread. The epidemiological triad consists of 1) agent (in this case, a pandemogen), 2) host (susceptible population or individuals), and 3) environment (extrinsic factors that affect the agent and opportunity for exposure). Main strategies to reduce people's chances of encountering a pandemogen are controlling 1) movement and mixing, 2) relevant behaviors, 3) builtenvironment, and 4) where applicable, disease vectors (beyond human-to-human transmission). Examples of NPI were drawn from the epidemiological literature and worldwide news reports of strategies used for covid during the first 18 months of the pandemic.

The classification scheme that resulted from iteratively matching principles with practices is outlined in FIGURE 1.

1000 Community control

- To restrict who can enter a community; to control access; to exclude individuals who are or might be infected

1100 Access to community; international \& internal borders

1110 Access to country; international borders

1120 Access to state, province, region; internal borders

1130 Access to other community defined by geographic area

1200 Access to structures

1210 Category of structure

1211 Type of structure

1300 Access to transportation services

1310 Category of transportation service 
1311 Type of transportation service

2000 Moving \& mixing

- To sequester communities (ring-fencing) or individuals (isolation and quarantine), to limit individuals' movement within a community, and to restrict mixing with other people with whom they do not cohabit

2100 Sequestration

2110 Ring-fencing, community

2111 Ring-fencing area

2112 Ring-fencing structure

2120 Isolation/quarantine, individuals

2121 At home, in facility

2122 Within facility

2130 Preventive sequestration

2200 Closure

2210 Category of structure closed

2211 Type of structure closed

2300 Stay-at-home order

2310 Category of stay-at-home order

2311 Type of stay-at-home order

2400 Mixing restriction

2410 Category of restriction

2411 Type of restriction

3000 Testing \& tracing

- To identify individuals to isolate, to quarantine, or to exclude; for pandemic response management

3100 Mass testing

3110 Population testing

3111 Ring-fence testing

3112 Community testing

3120 Surveillance testing 
3121 Type of structure or establishment

3200 Access control testing

3210 Category of structure or establishment

3211 Category/type of structure or establishment

3300 Case tracing

3310 Traditional case tracing

3320 Digital case tracing

3321 Smart-phone app

3322 Digital registration

3400 Symptom self-checking \& reporting

3500 Epidemiological testing

3510 For pandemogen (or other evidence of infection)

3520 For antibodies (or other evidence of immunity)

3530 For pandemogen genome (eg, to track variants)

3600 Proxy testing (eg, waste water analysis)

4000 Personal performance

- Individuals' specific behaviors to protect themselves from being infected, or, if infected, to avoid spreading the infection to other people.

4100 Personal hygiene

4110 Category of hygiene

4111 Type of hygiene

4200 Personal conduct

4120 Category of conduct (including socio-physical distancing)

4121 Type of conduct

4300 Personal warning (to be able to avoid contact)

4310 Warning by infected individual

4311 Physical warning (eg, bell, cane)

4312 Digital-age warning (eg, from smart-phone) 
4320 (Digital-age) Warning about (heavily infected) area

4321 Country (international)

4322 Region, other internal (national) area

4323 Locality hot-spots

4330 (Digital-age) Warning about (heavily infected) structure

5000 Environmental engineering

- To reduce chances of spreading pathogens within built environments through construction and maintenance and environmental monitoring and cleaning

5100 Environmental constructions

5110 Central ventilation (HVAC) systems \& certifications

5120 Touch points

5130 Socio-physical distancing

5200 Environment conduct

5210 Ventilation-related

5220 Environmental cleaning

5230 Shared item (removal)

6000 Bodies \& burials

- To protect the living from the dead

6100 Managing the dead

6110 Handling bodies

6120 Dealing with possessions

6130 Disinfecting accommodations, occupied spaces

6200 Burials

7000 Infection interdiction

- To reduce vectors' access to humans in order to prevent or slow pathogen transmission; vary by disease/vector

8000 NPI not elsewhere classified

Figure 1: NPI classification scheme; (sub)branches have potential subclassification 'not elsewhere classified.' 


\section{RESULTS}

For classification purposes, NPI can be organized according to the following broad categories: 1) community control, 2) moving and mixing, 3) testing and tracing, 4) personal performance, 5) environmental engineering, 6) bodies and burials, 7) infection interdiction, and 8) NPI not elsewhere classified. The following text describes each category and provides examples to illustrate types of NPI encompassed; they are not intended to be comprehensive. Popular terms that have various or vague definitions are not used in the NPI classification scheme, but may be used to explain a particular classification. Further, while examples may apply to covid, the scheme applies to NPI used to control any pandemic.

\subsection{Community Control (Figure section 1000)}

3.1.1 Overview: The goal of community control is to restrict who can enter a community; to control access or to exclude individuals who are or might be infected (including carriers of the disease). Essentially, there is a controlled boundary between a population and a defined community to limit people's movement, both into (and in some circumstances, out of) the community. Community may be defined, eg, by area, structure, or transportation service. Examples include 1) international border controls, 2) access to structures, and 3) boarding transportation services such as airline flights. Policies may include complete block of entry or conditional entry, as the following examples illustrate. Such screening may have consequences beyond exclusion. For example, international travelers who on arrival test positive for a pandemogen may be involuntarily institutionalized in an isolation facility and subject to monetary or other penalties.

3.1.2 International \& internal borders: When the community is a country, international borders may be closed or controlled. For example, travelers from all or specified countries may be excluded entirely. Individuals may be granted entry if they meet certain conditions, such as 1) period in quarantine (eg, in a government-facility, at home), 2) negative pandemogen test by an accredited laboratory (eg, on arrival, no older than 48 hours before departure), 3) agree to use a smart-phone app to report their health status to authorities daily but not be required to self-quarantine, or 4) any combination of these or similar strategies. In some circumstances, individuals living in a country may be required to obtain government permission to leave if they want to return because quarantine facilities have limited capacity (so that a valid exit permit becomes a condition for entry).

3.1.3 Access to structures: The permanent or temporary population of a structure is regarded as a community. Structures, include factories, office buildings, restaurants, retail establishments, and indoor and outdoor event venues. Access to the community can be controlled and potentially infected people excluded. For example, people who want to enter an office building (workers, suppliers, visitors, etc) may have to show evidence that they are not infected. Such evidence may include 1) vaccination/health passport, 2) negative pandemogen test, 3) normal temperature at entry, 4) answers to a self-administered questionnaire, or 5) any combination of these or similar strategies. The maximum number of people permitted to be in a structure at any one moment may also be specified (necessitating entry controls and exit monitoring). Further, clients of or visitors to a 
structure may have to register their contact information (when they would ordinarily not have to do so) in order to gain entry (to facilitate contact tracing).

3.1.4 Access to transportation services: An airline, eg, may require passengers to show evidence of non-infection in order to be permitted to board a flight. Further, access to flights requires access to an airport, a structure (considered above). Various types, or combinations, of evidence may be required.

\subsection{Moving \& Mixing (Figure section 2000)}

3.2.1 Overview: Moving and mixing refers to a set of policies and strategies that are intend to sequester communities (ring-fencing) or individuals (isolation and quarantine), to limit individuals' movement within a community (sometimes referred to loosely as lockdowns), and to restrict mixing with other people with whom they do not cohabit. Limiting movement may itself restrict people's opportunities to interact (eg, on public transportation). The main strategies in the set are 1) sequestration, 2) closures, 3) stay-at-home orders, and 4) mixing restrictions.

3.2.2 Sequestration: Sequestration involves isolating communities or individuals from the general population. Sequestration may be complemented by testing. An individual's immunological status (eg, based on vaccination, surviving documented infection, or presence of active antibodies) may affect sequestration implementation.

3.2.3 Ring-fencing: Perimeters may be established around geographic areas or structures (eg, apartment blocks) in which infected people are living in order to prevent them from leaving and noninfected people from entering. Such ring-fencing may be virtual (ie, informing people not to leave or to enter an area or structure) or physical (eg, barriers surrounding an area or structure and armed guards to enforce sequestration). Movement within a sequestrated area or structure may spread the pandemogen so that a local stay-at-home order may also be issued. Within a structure, a pandemogen may spread through a shared ventilation system; necessitating a prolonged period before the ringfencing perimeter can safely be removed. If a town, eg, erects barriers to prevent infected people from entering, such strategy is considered to be access control.

3.2.4 Isolation/quarantine: An individual may be instructed to self-isolate/quarantine at home or may be admitted to an isolation/quarantine facility. Admission to a facility may be required when 1) an individual is homeless or whose home is unsuitable, 2) involuntary commitment is required (eg, because the person breaks self-isolation), or 3) border control or other special circumstance. An isolation/quarantine facility may be a purpose-built facility (with proper ventilation and internal infection control systems) or a requisitioned structure (eg, quarantine hotel in which to house international arrivals). Various digital-age technologies may be used to monitor or to help to enforce isolation/quarantine (eg, electric ankle bracelets, smart-phone apps). Within an isolation/quarantine facility, infected individuals may be housed separately from those in quarantine. Within a structure, infected individuals and those who may be incubating the disease may be sequestered from the rest of the population (eg, in hospitals, care homes, and prisons and other congregate living structures). 
Such sequestration may involve physically moving individuals from one section to another or, if housed in separate, appropriately equipped rooms, instituting a higher level of infection control when infection is confirmed or suspected.

3.2.5 Preventive sequestration: The goal of preventive sequestration is to prevent populations from mixing; from becoming infected, particularly essential workers. Types of preventive sequestration include: 1) pandemic dormitories (eg, for workers), 2) pandemic bubbles (eg, for school children), and 3) pandemic pods (eg, for limited social interaction). Hospital workers may be housed at the worksite or in near-by requisitioned hotels in order to sequester them from the general population, thereby reducing chances of them contracting the disease at home or while traveling to and from work (or transmitting it to others at the worksite, while traveling, or at home). Such pandemic dormitories may have their own isolation/quarantine sections. The temporary population of a structure may be divided into small groups (eg, school children by grade), with each group kept physically separate from arrival to departure (ie, occupying a bubble). Where permitted, a pandemic pod can be formed, eg, by a small number of families, in order to permit members to interact, particularly children for educational purposes, while reducing the risk of infection. From an NPI classification perspective, a travel bubble is created by differential border controls. For example, residents of 2 or more bubble countries may be free to travel among them (or with fewer restrictions), whereas those of non-bubble countries may be excluded or admitted conditionally.

3.2.6 Closures: Closures refers to the strategy of closing structures or services, and/or banning outdoors events, in order to prevent people from coming into close proximity with one another. Limiting capacity is considered to be a partial closure, ie, $0 \%$ capacity is complete closure; $100 \%$ capacity means open as usual. Closures may involve structures, services, or events. Some closures or restrictions may be defined by factors other than capacity. For example, some retail establishments may be allowed to stay open (within certain hours) but allowed only sell items that are considered to be essential; ditto bars, but not being permitted to serve alcohol. Examples of structures that may be ordered to close include 1) worksites (factories, offices, building sites) 2) educational establishments (eg, child-care centers, kindergartens, schools, universities), 3) restaurants and bars, 4) retail establishments (especially if considered to be non-essential), 5) gyms, swimming pools, and other recreational and community facilities, 6) places of entertainment (eg, cinemas, theaters, nightclubs, museums, art galleries, sports arenas), 7) places of worship, 8) exhibitions and conventions, and 9) outdoor events (eg, music festivals, mass demonstrations). Services that may be ordered to close include local, long-distance, or point-to-point public transportation systems. Capacity limitations are considered to be partial closures. For example, restaurants may be allowed to open but limit indoor dinners to a maximum of $25 \%$ of permitted capacity. Examples of functional limitations include being allowed 1) to be open only certain days or hours, 2) to serve only diners when seated outdoors, and 3) to offer only take-out meals. Restrictions on businesses may include 1) work-from-home orders, 2) on-line (instead of in-person) interactions and meetings, and 3) limiting the number of persons permitted in business meetings. Outdoor events may be limited by maximum number of attendees; the number reflecting the perceived risk of spreading the pandemogen among them. Socio-physical distancing may also be required; described later. 
3.2.7 Stay-at-home orders: Residential confinement applies principally to people's housing, including such congregate living facilities as dormitories, group homes, and half-way houses. The goal is to limit movement from home in order to limit contact with other individuals or mixing among people, thereby reducing chances for spreading the pandemogen. Stay-at-home orders (SAHO) are rarely a complete prohibition of leaving home due to the difficulty of supplying people. Such order is different from isolating or quarantining at home, which may involve a complete prohibition on leaving home for a specified period. A SAHO may grant automatic or conditional permission to leave home. For example, usual types of automatic provision include 1) allowing one household member to go shopping once per week (who may or may not have to register with authorities for enforcement purposes) and 2) attending medical appointments. Exceptions may also apply for essential workers when traveling to and from or for work-related purposes. People may be allowed to request permission from authorities to leave home for a specified period, eg, to attend a funeral (and when out-and-about may be required to show evidence of such permission). Alternatively, a SAHO may only specify restrictions on leaving home, including curfews. For example, people are free 1) to leave home but are permitted to move only within a 5 kilometer perimeter or 2) to go out except to walk-the-dog. Curfews are considered to be a type of enforced residential confinement delineated by time of day. Limitations on the use of private transportation are considered to fall within SAHO. Choosing to shield-at-home voluntarily is considered to fall within personal performance.

3.2.8 Mixing restrictions: Mixing restrictions are NPI that are intended to limit in-person gatherings of people who do not cohabit. For example, a government order may prohibit or restrict gatherings (such as going out to or meeting at a restaurant, birthday parties, backyard barbecues, eg, limiting any such permitted event to no more than three families and no more than a total of 6 people.

\subsection{Testing \& Tracing (Figure section 3000)}

3.3.1 Overview: In pandemics, various types of tests and testing may serve different objectives, including 1) identifying individuals to be isolated or quarantined, 2) controlling access, and 3) conducting epidemiological surveillance (including testing waste-water to identify communities with a seemingly high rate of infection in order to target mass testing). Testing may be performed by public health, worksite, or service personnel or by individuals at home (self-testing). Testing policies can be complex and difficult to implement. Issues include 1) if supply is limited, establishing priorities for who should be tested 2) making test results available within the decision horizon, 3) using tests that are sufficiently accurate for the purpose and, depending on why and how testing is being conducted, dealing with the consequences of false positives and false negatives, 4) setting up testing stations to minimize chances that infected individuals can spread the pandemogen to testing staff or others waiting to be tested, and 5) assuring that population testing does not unduly strain health services. Testing and tracing are intertwined: testing can facilitate contact tracing but tracing does not depend on testing. The goal is to identify individuals who are infected (so that they can be isolated) and those who have been in, or might have come into, contact with an infected person (so that they can be quarantined); also to exclude such individuals (for access control purposes). Tracing must be quick enough to make a meaningful difference to pandemogen spread. 
3.3.2 Mass testing: Mass testing can be divided into 1) population testing and 2) surveillance testing of individuals. While population testing may involve teams swooping in and testing everyone and then departing, surveillance testing may involve a dedicated team within a structure. Population testing may be defined by 1) ring-fence testing (in which everyone within an area or structure is tested) and 2) community testing (in which testing stations are established within a community). Testing stations may be 1) mobile facilities or 2) temporary structures (eg, tents) or requisitioned buildings; for drive-through or walk-through testing. Existing health care facilities, eg, hospital emergency departments, can function as ongoing testing stations. Surveillance testing may typically involve worksites but can also be employed in other structures (eg, for patients in care homes). Within a work place, surveillance testing may involve ongoing testing of all or samples of workers (located within or associated with the worksite); not only to identify infected individuals but also to decide if infection controls should be strengthened or if it should be closed due to the rate of infections. In sensitive situations, testing of all workers may be done daily.

3.3.3 Access control testing: Testing for purposes of controlling access to an area or structure may be done 1) prior to arrival (in which case a suitable test certificate must be presented) or 2) on site at time of access (if sufficiently accurate, practical tests are available for the purpose). For example, schools may employ on site access testing to decide who can attend school that day (with students testing positive sent home to self-isolate).

3.3.4 Case tracing: Case tracking comes in 2 main flavors, which can be used simultaneously. They are 1) traditional case tracing (which is facilitated by but does not rely on testing) and 2) 3Tstrategy of community mass testing developed for covid. The 3T-strategy involves 1) preemptive population-testing, 2) prompt tracing of infected individuals and their known contacts, and 3) appropriate treatment or follow up. Case tracing as a means of suppressing a pandemogen is only practical when the number of cases is relatively small. It involves 1) tracing contacts of people who test positive for (or exhibit signs or symptoms of) the disease, 2) determining if a contact is infected, and 3) for positives or symptomatics, isolating these individuals and tracing their contacts; or otherwise quarantining traced individuals (and tracing the contacts of isolated individuals and those who develop the disease during quarantine). If an individual is ordered to self-isolate at home, the advice for cohabitants is to maintain as much distance between that infected individual and other members of the household as practical. The alternative is to isolated the infected person in an isolation facility, either by policy or if the home is unsuitable, or, possibly, by individual preference. The same logic can be broadened to apply to quarantine. The obvious disadvantage of case tracing when testing is not available is that it misses asymptomatic cases; also individuals may be confined longer than would otherwise be necessary. Digital-age contact tracing can augment tradition case tracing. It can entail 1) smart-phone digital-app or 2) digital registration (eg, to access a restaurant). It involves 1) keeping track of everyone with whom an individual came into contact and/or was close to for more than a specified number of minutes and 2) if the digital-app user (or any captured closecontact) tests positive within a specified period after contact, alerting the user (or close-contact) to self-quarantine and/or to get tested. Poorly designed digital-apps can generate a pingdemic, when large numbers of individuals are advised unnecessarily to self-isolate or -quarantine, creating worker shortages (and undermining confidence in public health authorities). Additionally, AI-analytics can be used to trace people, eg, using facial recognition or credit card charges. 


\subsection{Personal Performance (Figure section 4000)}

3.4.1 Overview: Personal performance NPI are specific behaviors intended to protect individuals from being infected, or, if infected, from passing the infection to other people. They depend on individuals performing them, even when mandatory. Such NPI can be divided into 1) personal hygiene, 2) personal conduct, and 3) personal warnings. Behaviors are understood to have been ordered or recommended by public health authorities. Consequently, in the classification scheme, if public toilets have been ordered closed, eg, that classification would be considered to be determinant (although any recommendation to avoid use of public toilets could still be coded separately). Some NPI when conducted at home are considered under environmental engineering, the home being the structure that is engineered. Self-monitoring for symptoms of infections (eg, using a digital-age symptom-checker) is considered to be self-testing and is classified under testing and tracing.

3.4.2 Personal hygiene: Personal hygiene strategies include: 1) hand-washing and/or sanitizing, 2) using mouthwash, 3) avoiding spitting and uncovered sneezing and coughing, and 4) using appropriate personal protective equipment, specifically masks (and, when appropriate, face shields, goggles, medical gowns/caps, helmets, bubbles, hazmat suits, gas-masks, respirators, self-contained breathing apparatus, etc).

3.4.3 Personal conduct: Personal contact strategies may involve socio-physical distancing. Such strategies include 1) shielding at home, 2) staying home when sick or displaying symptoms (especially of the pandemogen), 3) when out and about, maintaining a specified distance from other people (eg, 2 meters), 4) avoiding personal interactions (eg, no hand-shaking, no hugging, no kissing), 5) moderating exercise in order to reduce pandemogen shedding, 6) refraining from exchanging items, 7) avoiding pushing buttons, etc (eg, by using no-touch technology), 8) preferring no-contact services (shopping online, curb-side pickup, delivery service, etc to minimize in-person contact), and 9) observing hot-spot and other warnings. Some personal conduct NPI involve structures which are subject to closures or restrictions. Examples include 1) working from home, 2) limiting time spent indoors in spaces shared with other people (eg, bars and restaurants), 3) avoiding contact, and/or limiting time spent, with non-household members, indoors and outdoors, 4) avoiding large gatherings (eg, conventions, sporting events, festivals), and 5) avoiding use of public toilets/restrooms.

3.4.4 Personal warnings: Personal warnings tell people about individuals, structures, or places that should be avoided due to reported infections. Warnings may be physical or digital. Infected individuals when moving about, in biblical times, were required to wear a bell and, in the middleages, to carry a white cane. In the digital-age, websites and/or communications to smart-phones may indicate 1) hot-spots, communities or places where an unusually high number of infected individual have been found or 2) countries with a high rate of infection (which ratings and warnings may be expressed as traffic-light colors and have policy consequences such as mandatory travel restrictions). Personal alerts about having been in contact with an infected individual are considered under testing and tracing. 


\subsection{Environmental Engineering (Figure Section 5000)}

3.5.1 Overview: Environmental engineering strategies reduce chances of spreading pandemogens within built environments through construction and maintenance and environmental monitoring and cleaning. They can be divided into 1) construction and 2) conduct.

3.5.2 Environmental construction: Environmental construction strategies include 1) central ventilation systems, 2) touch-points, and 3) socio-physical distancing. Central ventilation system can be improved (eg, by using positive pressure air-flow systems, upgrading ducts to improve air flows, increasing air exchanges, adding filters); possibly, when meeting required standards, being certified. Touch-points include 1) switching to microbe-killing surfaces, and 2) replacing physical buttons, door pulls and pushes, access cards, etc with no-touch technologies. Social-physical distancing considerations include 1) strengthening entrances to be able to control better the number of people in the structure at any one time, 2) installing partitions, screens, etc (between service providers and customers, between tables in restaurants, etc), 3) constructing one way people flows (entering at one door, move in one direction, and exiting at a separate door), to maximize distancing and to minimize mixing, 4) marking floors, chairs, etc to facilitate people keeping physical distance from each other, 5) creating signage to remind people about socio-physical distancing, 6) deinstalling buffets and other areas in which people would ordinarily mix.

3.5.3 Environmental conduct: Environmental conduct strategies include 1) ventilation-related changes, 2) environmental cleaning, and 3) removing shared items, including substituting single-use items. Ventilation-related changes include 1) stilling air flows to avoid circulating pandemogens (eg, by switching of air-flow hand dryers in toilets and fans in medical examination rooms), 2) adding portable HEPA filters and UV-lights to rooms, 3) opening windows to increase air-exchanges, and 4) installing carbon dioxide meters to indicate air-exchange quality (and when it is approaching the danger zone). Environmental cleaning examples include 1) routine, vigorous cleaning of spaces and surfaces, 2) routine cleaning of touch points (eg, door-pushes, door-handles, push-buttons), and 3) sanitizing incoming/exchanged items.

\subsection{Bodies \& Burials (Figure section 6000)}

Protecting the living from the dead involves 1) managing the dead and 2) arranging burials or otherwise disposing of bodies. Managing the dead includes 1) handling bodies of people who died, or might have died, of or with the pandemic disease (when handling the body can spread the disease), 2) dealing with their possessions, and 3) disinfecting their accommodations or occupied spaces.

\subsection{Infection Interdiction (Figure section 7000)}

A pandemogen may be spread by human to human transmission, either directly (eg, breathing on another person, sexual intercourse) or delayed (eg, transmission through air-circulation, food, water, objects, surfaces, debris, medical devices, dead bodies). Mode of transmission can also be classified, eg, aerosols, fluids, fomites). Some pandemogens are also spread by vectors (think plague, zika). 
Vector transmission may be classified as mechanical or biological. Reducing vectors' access to humans may slow transmission. Such NPI would be classified under infection interdiction. They vary by disease.

\subsection{NPI Not Elsewhere Classified (Figure section 8000)}

This category is a catchall for any NPI that cannot be classified according to substantive categories. The expectations is that as the scheme evolves any such NPI 1) could be classified according to an existing category, 2) would lead to the creation of an additional category, or 3) would require reconfiguration of the classification scheme.

\subsection{Coding \& Using NPI}

3.9.1 Coding design: Coding NPI for informatics and other purposes is based on a cube design: 1) type of NPI, 2) policy characteristics, and 3) space-time (place and period NPI employed). The second dimension captures pertinent policy characteristics associated with an employed NPI. Such characteristics may include: 1) force with which employed, 2) enforcement, 3) to whom the NPI applies, and 4) how it is employed. For example, force may be characterized as mandatory or voluntary. Further, variability in policy can be captured as specific variables when such characterization is needed but greater granularity is not. Structured codes describe a unique position in the cube which when captured in a relational database to provide a powerful analytic tool. Where they exist, the scheme uses standard code sets (eg, to identify countries); including flavors of null. Where they do not, needed code sets could be developed as part of an international standard and, if applicable, used beyond NPI classification. The goal is to code to the level required for the purpose (although the scheme allows NPI to be described at a granular level). For a coded NPI, the scheme also permits capturing details in unstructured text (and, if there are many such records, analyzed using natural language processing). Such detail may include specific NPI requirements or exceptions (eg, essential workers exempted from stay-at-home orders). Further, if a country's border controls require travelers to be quarantined in a government-run facility and such capacity is limited, the country may restrict the number of international flights arriving each day. Such natural consequence could be captured as unstructured data (rather than as a separate NPI record).

3.9.2 Using NPI \& machine learning: Improving pandemic preparedness and future PRM depends on being able to learn systematically from experience in responding to the covid pandemic. The classification scheme allows NPI employed in responding to covid 1) to be described using a common structure in as much granularity as needed (type of NPI, start and end dates, location, force with which employed, etc) and 2) to be compared meaningfully across countries and regions within them (at whatever level of granularity was commonly encoded); including changes in the mix of NPI employed as the pandemic unfolded. Such structured NPI data can also be correlated with pertinent contextual background, infrastructure, and situational variables to gain insights into the dynamics of PRM and the effectiveness of various combinations of policies and strategies (PASS). Structured NPI data - when applicable, augmented with data on vaccine rollouts - can be used as inputs to machine learning. Such predictive models can potentially be used 1) to discover hidden insights, 2) to predict such outcomes as death rate, economic activity, and public sentiment, and 3) possibly, to 
suggest the optimal mix of NPI/PASS depending on contextual background and evolving situation on the ground at different stage of a pandemic. In the future, AI analytics can be expected to play an ever-expanding role in guiding PRM. Moreover, machine learning may also prove to be useful for refining the NPI classification scheme itself.

\section{DISCUSSION}

This article proposes a first-ever NPI classification scheme; filling an important gap and building an initial inventory of types of NPI. It is not intended to be a fully-elaborated classification scheme nor to be a complete inventory of NPI. Classification facilitates 1) describing and analyzing NPI (eg, comparing how countries used different NPI to respond to covid), 2) evaluating NPI effectiveness, and 3) using machine learning to identify optimal combinations of NPI for responding to pandemics as they unfold). Classification does not reduce the need for clarity in implementation. For example, when implementing NPI, governments need clear rationale for and definitions of such collateral concepts as essential workers and non-essential businesses. The utility of any classification scheme depends on its acceptability by target users. Where primary users are located throughout the world, the development of an international standard may promote its use. The standards development process would allow experts from different backgrounds and with various perspectives to refine the classification scheme to enhance its utility. Worldwide use of the resultant classification scheme 1) would aid describing and comparing PRM interventions across countries, 2) would allow standardization of data analyses, and 3) would facilitate use of results in policy decision-making. Best next steps may include 1) elaborating the classification scheme as an international standard, 2) specifying the corresponding coding structure in operation detail, and 3) maintaining a corresponding set of standard definitions. The standard would be reviewed periodically and revised to keep pace with advances in NPI, their use in practice (such as in machine learning), and results of policies and practices pertaining to their use.

\section{CONCLUSIONS}

The NPI classification scheme presented here is an important step forward in consistently and reliably describing and analyzing NPI, comparing results internationally, and facilitating the use of real world data to improve the management of future pandemics. This scheme could serve as the basis for developing an international standard toward promoting worldwide a common understanding of NPI and a uniform way of classifying them. Once developed such standard could serve as the basis for the development of a corresponding ontology. In the interim, any entity could apply the NPI classification scheme described here to suit its purposes.

\section{CONFLICTS OF INTEREST}

None 


\section{ACKNOWLEDGMENTS}

None

\section{References}

[1] https://www.medscape.com/viewarticle/940788

[2] https://www.economist.com/search?q=The+pandemic $\% \mathrm{E} 2 \% 80 \% 99 \mathrm{~s}+$ true + death+toll.+www. economist.com $\% 3 \mathrm{~B}+$ December $+22 \% 2 \mathrm{C}+2021 . \&$ sort $=$ date

[3] Ibrahim MD, Binofai FAS, Aishams RMM. Pandemic Response Management Framework Based on Efficiency of COVID-19 Control and Treatment. Future Virology. 2021;15:801-806. 